\title{
Exploring the Capabilities of Industrial Collaborative Robot Applications
}

\author{
Staffan K.L. ANDERSSON ${ }^{\mathrm{a}, 1}$, Anna GRANLUND ${ }^{\mathrm{a}}$, Mikael HEDELIND ${ }^{\mathrm{a}}$, Jessica \\ BRUCH $^{\mathrm{a}}$ \\ ${ }^{a}$ Department of Innovation and Product Realization, Mälardalen University, Sweden
}

\begin{abstract}
The increase in customization is pushing companies to use more advanced automation technologies in their production lines. Yet, assembly operations are predominantly performed by humans because of their ability to be flexible. The emergence of industrial collaborative robots provides an opportunity to have robots work alongside humans in a flexible and collaborative application. The aim of this study is to explore the industrial collaborative robot capabilities in a collaborative application compared to traditional robot applications. This interview study draws data from four companies with experience in industrial collaborative robot applications. The companies involved in this study experienced that there are several benefits of using an industrial collaborative robot but challenges still exist, in particular related to usability and the robot integration process.
\end{abstract}

Keywords. Industrial Collaborative Robot, Capabilities, Human-robot collaboration, Interview study

\section{Introduction}

Increasing demand of customized products pushes companies towards increased flexibility when adapting products to each customer. Advanced automation have been known to be an enabler of such flexibility [1]. In manufacturing, advanced automation such as industrial robots have traditionally been employed to handle repetitive and dangerous tasks [2]. Assembly operations, on the other hand, are still highly reliant on humans where they are the predominant flexibility enabler. Thus, assembly operations are generally designed for manual product assembly [3]. Until recently, there have been few ways to match the human capabilities paramount for assembly operations, but the emergence of industrial collaborative robots (ICR) have provided opportunities to introduce robots even in assembly operations [4-7]. ICR applications provides an opportunity to combine the decision making and intuition of the human with the speed, accuracy and strength of the robot to achieve a flexible application $[6,8]$. Yet, to this day there are few companies that have integrated the ICR applications [9]. Partly because companies are still investigating the capabilities of the ICR application to understand what benefits can be gained with this type of application. ICRs are one of the drivers for the expected increase in industrial robot investments the coming years [10]. The investments in ICR are expected to grow extensively the coming years where assembly, material handling and pick and placing are expected to denote $50 \%$ of the applications [11]. This development descends foremost from a rise in labour cost and simultaneously

\footnotetext{
${ }^{1}$ Staffan.Andersson@mdh.se
} 
decreasing robot costs [10]. The purpose of this paper is to explore the capabilities of industrial collaborative robot applications, compared to traditional robot applications.

For the purpose of this paper, ICR concern the collaborative robot in itself and the ICR application encompass the shop floor application including operator, robot and related equipment in the workstation where the operator and ICR are working towards the same goal. ICR are sometimes mentioned as CoBot by the companies.

\section{Methodology}

This interview study was conducted on the premises of exploring companies that are currently using, or are in the pilot phase prior to installing, an ICR application. Findings from the interview study emerged from a thematic analysis.

\subsection{Interview study}

In-depth interviews were carried out between Mars 2019 and October 2019 with managers and engineers involved with the development and daily operations of ICR applications. Firstly, the focus was on mapping the companies' experiences of traditional robots. Thereafter, it was investigated how the companies worked with the ICR application. Interview questions covered the following areas; the differences between traditional robots and ICR, what companies had learned during the integration of an ICR application, and the how the capabilities of the ICR were utilised. Three of the companies are in automotive industry, both component manufacturing and final assembly operations are represented. Additionally, one company is a manufacturer of high volume, high variety products. Two of the companies have integrated ICR in their production line.

To fulfil the aim of this study, two of the interviews were carried out with academics working closely with development of ICR applications. Hence, these two interviews represents both an academic and a company perspective regarding the ICRs capacities in collaborative applications. It was identified that two of the case companies have pilot areas where the ICRs are being tested in a lab environment. However, the pilots are closely representing the real world application. Two out of four companies have ICR applications in the production line.

\subsection{Data Analysis}

A structured deconstruction of data was undertaken, starting with reading trough the interviews as a whole, ending up with condensing the data into a set of codes. The coding was based on the steps provided by Braun and Clarke [12]. These codes were then expanded to rich descriptions about the ICR capabilities and related challenges faced by the companies. The codes consisted of capabilities, reasons, and traditional robot. Capabilities focused on how the ICRs are utilised and reasons addressed why an ICR was selected in the first place. Traditional robot was applied in order to identify how the companies differentiate between the ICR and traditional robots. Finally, challenges focused on the experiences related to the difficulties in the capabilities often compared to traditional robots, or other automation equipment, when applicable. 


\section{Concerning ICR applications and capabilities}

This section explores the key capabilities of ICR by analysing case studies in previous research where ICR applications have been employed in industry or in experiments.

\subsection{Defining ICR application}

There exists a wide range of robots capable of collaborative applications spanning from medical applications, supportive exoskeletons and human-like robots [13]. In turn, ICRs encompass lightweight robots that are used in an industrial setting. The ICR application include a robot that complements the flexibility and cognitive skills of the human with repeatability and robustness of the robot [5]. ICRs are commonly used in assembly operations working jointly with a human, thus forming an ICR application which is a "state in which a purposely designed robot system and an operator work on simultaneous tasks within a collaborative workspace" [14, p. 2]. This one dimensional description need to be complemented with a higher resolution on collaboration due to the fact that many different applications exist in a variety of contexts.

The proposed framework by Aaltonen et al. [15] aids to define the collaboration levels between robot and operator. The four top levels presented for collaboration are: no Coexistence, Coexistence, Cooperation and Collaboration. No Coexisting are fenced up robots, Coexistence means that human and robot have separate activities but work in close proximity to one another, Cooperation is similar to ISO 10218 [14] as stated above, and finally Collaboration means the ICR and operator are sharing both tasks and space. Each of these levels include sub levels addressing, for instance, if contact is allowed between the robot and operator. Nonetheless, in the ICR application the companies need to adhere to the machine directive [16] to uphold the safety standards in general and the collaborative robot standard specifically [17].

In a literature review in 2019, Hentout et al. [4] compared ICR with traditional robots by providing a mapping of the outstanding differences relying on the notion that all traditional robots are indeed heavy and unsafe for collaboration compared to the lightweight and inherently safe ICRs. On the other hand, large traditional robots can be used in collaborative applications [18] supported by monitoring systems that combines technologies such as sensors, vison systems and laser scanners thus, achieving safety in collaborative applications [19].

\subsection{ICRs in industry}

In a multiple-case study by Bauer et al. [20], only twenty five integrated ICR applications were identified in industry, and some of the applications were still in a state of demonstration. In academia, the industry-applied cases are furthermore limited to a few papers (see for example [18-23]) this emphasizes the need to define the ICR capabilities and the benefits of using an ICR application. Moreover, companies need understand why and how to integrate this automation technology, which is considered a challenge in industry [24]. As suggested by Land et al. [25], companies that are considering to use a ICR application are hoping to achieve ergonomically beneficial and cost reducing applications.

In the early works of Akella et al. in 1999 [26] they introduced a weight supporting robot to increase flexibility and productivity providing one of the widely known 
integrations of ICR applications. Flexibility in ICR applications means that the application is adaptable to variations in the process or product, which can be realized by, for instance, external cameras and sensors [27].

The work of Guerin et al. [22] proposed that flexibility can be possible, even for small and medium sized enterprises due to the fact that some ICRs are easy to program and robust even when the application is rearranged. By introducing easy programming, companies that have limited experience with automation have the potential to employ ICR applications [19, 20]. Ease of programming is nonetheless dependant on the programs used. Moreover, there is a need for the applications to be robust even when there are changes in production [23] and robustness can be achieved with the simple programming $[20,28]$. In addition, the ICRs have been recognized to sometimes be more flexible than traditional robots because the lightweight ICRs can be swiftly rearranged to a new application [29].

In a case study in 2019, Faccio et al. [30] provided a comparison model for evaluating ICR application and non-collaborative automation where they found that throughput might suffer in the ICR application. Moreover, in their study the case company had an ICR with a screwdriver integrated in the end effector; however, little considerations were made regarding the safety concerns for the pointy and sharp equipment attached to the robot. Safety is still one of the major concerns when dealing with ICR applications [9]. Furthermore, sharp objects might results in an application that is dangerous and safety might not be guaranteed.

\section{Capabilities - Empirical Findings}

In this section, the experiences from the companies are presented, focusing on the ICR capabilities flexibility, safety and applicability. The respective ICR applications are described firstly as to define the context and provided an understanding of what reasons the companies had to integrate or investigate the ICR applications. The companies in this study are ICR application end-users.

The companies in this study had different reasons for integrating the ICR. One company expressed that the need originated from a desire to test and evaluate new technology, namely ICR, while simultaneously reducing the cost in the assembly operations by removing the need for an operator in one of the stations. The station was considered a bottleneck because the operator needed to move between stations thus resulting in an increased cycle time. In the employed ICR application, the operator and the lightweight dual-arm ICR work in separate stations and the ICR is sometimes stopped when the operator need to work within the ICRs workspace. While the operator is feeding the station, the ICR is working. This ICR application concerns collaboration on a coexisting level where contact is only allowed to a small extent.

Another company integrated an ICR because they needed repeatability, robustness and high availability in a material handling station. In this station, the ICR need to pick small components precisely with two arms. The dexterity required for this station was considered to be difficult for a larger traditional robot. This company used the same robot as the company above. Namely, a two armed light weight robot.

The two last companies are at the pilot stage of integration, hence have not yet integrated the ICRs in their production line. The ICR used in these applications are onearmed non-padded robots with collaborative capabilities such as force limiting. Both companies presents ergonomic improvements as one of the main reasons for using an 
ICR. Also, increased productivity and flexibility are desired capabilities from the ICR. Nonetheless, both companies identifies, that there are currently several challenges to be addressed before these capabilities can be achieved.

\subsection{Flexibility}

The desired capabilities for flexibility varies between the companies and only two of the companies dedicated flexibility to be a desired capability. One company stressed that portability was a capability for flexibility since they wanted to be able to move the ICR swiftly between stations if needed. In their experience, the current traditional robots that are employed in the production area are immovable and difficult to re-locate because the robots are fenced up and mounted to the floor. The company also identified that their maintenance personnel found the ICR easier to program than the traditional robots because the ICR had a program made specifically for that type of robot. The programing made it simpler to make robust programs swiftly via lead-trough programing. However, the competences needed to program the ICR was recognised as a challenge in the early phases of the integration process because the company did not possess these specific competences.

A second company views the ICR as more flexible than traditional robots because the ICR is believed to be able to manage an increase in product variety thanks to easy programming. It was nonetheless recognised that to manage such programming there would be a need to educate both operators and maintenance personnel on the ICR software.

\subsection{Safety}

When it comes to the safety in the ICR applications, the companies have different approaches to manage this aspect. Naturally, safety is of great concern for all the companies but depending on their previous experience with robots, they have a variety of approaches on how to manage safety in ICR applications. When integrating the ICR, one company use the identical safety assessment for the ICR application as for other robot applications, in which the safety aspects are checked, fulfilled and approved for the attended application. As noted by one of the project members:

"[...] we just followed our normal safety assessment, it should be enough. We just did that until we checked everything. We feel confident about how we did that."

Because this company is experienced with traditional robot they found it convenient to assess the ICR application in a similar manner.

One of the companies is in pilot testing and lack experienced in robotics, believed that there are still major challenges concerning the safety. At this company the ICR application can be considered as non-portable at the current state due to the system complexity and challenges in safety assessment:

"The CoBot is used in an open environment and then you have to think more about what the safety will look like. There will be many changes along the way, how do you do that? For every 


\section{change, do you need to CE-mark and make a risk assessment every time? This is a challenge."}

The third company, that have experience with automation equipment, has a sober view on ICR application safety. Form their perspective much have been promised on ICR application safety as the applications are famous for being inherently safe. Hence, the company lowered their expectations on the ICR application by addressing several aspects that need to be evaluated in terms of safety:

\section{"[...] one has to take great consideration concerning the} safety and risk assessment in the whole [ICR] work-station. Is the gripper dangerous? The articles, are they soft or sharp? Even the surroundings. Is it a narrow space? Is there a risk of a visitor falling into the robot?"

Although safety is important for the operator working adjacent to the ICR, the context in which the ICR is placed must be evaluated as the robot itself might get damaged. These damages may occur when the ICR keeps working even when the production line has stopped. If the ICR continuous to work it risks getting wedge in existing equipment causing damages and further stoppages. This situation transpired at one company during the full scale production. Here, the ICR interacted with production equipment causing a situation where both the robot and equipment were at risk of damages. A production engineer explains this issue in their ICR application:

"You have to think about where you place it [the ICR]. If the line stops then the CoBot is unaware off this and continuous to work. If a machine door closes then the robot arm will still be there".

This situation occurred because of the type of ICR employed, the robot physically pushes acknowledgment buttons on the production equipment but is not connected to the production line interface, as the companies traditional robots are. To solve this issue, the ICR could to be connected to existing production software interfaces to be aware of production line disturbances. These statements points out that the safety aspect is context based, meaning that depending on the context - expertise, type of application, type of ICR, etc. - the safety needs to be amended to the situation at hand.

In conclusion, the safety is a challenge for the companies but different strategies to deal with the safety are employed. Companies that have dealt with automation previously have the benefit of already having safety assessments for automation in place. However, the ICR adds the complexity by an operator working adjacent to the robot, which arises a need to evaluate both the ICR and operator assembly operations for the ICR application.

\subsection{Applicability}

The assembly operations at the companies are exceedingly manual when it comes to material handling and parts assembly. Therefore, one of the reasons for using an ICR application is that the ICR can perform human-like assembly tasks such as lifting small parts and assembling with high precision. Human-like assembly is one of the capabilities that the one company identified as necessary to employ as the ICR application tasks were 
previously realised by an operator. Moreover, the ICR adds precision and repetitiveness commonly unreachable by operators.

Nonetheless, the companies experienced with traditional robots found that the ICR perform the operation considerably slower than expected from a traditional robot. The ICR was also identified to be slower than the operator previously performing the tasks. Additionally, evaluating the outcome of the ICR application is a challenge because the evaluation of the operator's performance encompasses many unmeasurable aspects, which a project manager exemplified by the following;

"The problem is that we don't know how long it would take to do the robot's work by hand. It depends a lot on who's doing it - if they are keen or tired, if the materials are in place. There are many variables to consider which makes it hard to compare"

The human-like assembly is therefore one of the desired capabilities, but challenges still exists in evaluating the capacity of the ICR application when comparing the application to an operator. Nonetheless, the ICR need to be programmed to be robust, which can be a challenge.

For instance, high accuracy and use of supporting functions, such as the built-in vision system in the investigated applications, can result in a situation where the ICR cannot meet the required cycle time, thus this capability is not used. This was expressed as a challenge by both a production and process engineer, respectively:

"There is an opportunity to set the CoBot up so that it sees where to assemble and everything. There is a camera in the wrist.

But then we exceed the cycle time, if you are too precise [...]. There are functionalities, but they take time. This is a problem when you have a short cycle time to consider".

Moreover;

"It was during the installation, when we adjusted it. We saw that this is taking too much time. There is a queue. I know we struggled with the cycle time before we were satisfied".

These issues derives from a lack of knowledge about the ICR application. The lack of knowledge caused the projects to be costly and time consuming partly because the ICR application did not reach the intended capacity during full production. In addition, because the capabilities were unexplored, the companies face difficulties when planning the ICR integration and specifying the ICR requirements in the initial stages of the projects.

\section{Discussion and Conclusions}

The purpose of this paper was to explore the capabilities of industrial collaborative robot applications, compared to traditional robot applications. This paper adds to existing knowledge on ICR application capabilities by presenting empirical examples from endusers. The findings in this study can be summarized into three main areas: flexibility, safety and applicability. Flexibility includes portability and the ICR capability of being 
easy to program. Safety concerns the assessments of safety, inherent safety and application safety. Lastly, applicability focuses on the capability of robustness, how the ICR capabilities can be evaluated and how the ICR performed in the intended application.

It is evident from previous research that the ICR have capabilities to be safe, easy to use and program, sensitive to the surroundings, adaptable, and capable of supporting the human in manufacturing processes $[5,6,9]$. The findings of this study exemplifies from the perspective of four companies with experience in ICR applications. This paper presents that the ICR have several capabilities that set it apart from traditional robots.

The findings in this study points towards that ICR applications have the capability of easier programming, especially compared to a traditional robot. Moreover, the ease of programming was a capability that the companies considered as a benefit. Portability was one of the preferred capabilities in this aspect, which Kock et al. [29] identified to set ICR apart from traditional robots. The companies expected that the ICR would be adaptable, but there is a need to reprogram the ICR due to process or task variations. In addition, there is an opportunity to configure and relocate the ICR to new stations thanks to this capability $[19,20]$. However, as emphasised by both the companies, Bauer et al. and Steinmetz and Weitschat $[20,28]$ that the application need to be robust, otherwise there might be risk of human injuries and damages to the ICR or other equipment.

From a safety perspective, the companies experience with automation felt confident using their existing assessment tools to verify the ICR application safety, but there are still concerns about how safety can be assured in ICR applications as there is a need to evaluate both operator and human. This points towards that safety assessment is depended on the end-users experience. A solution to this issue have been proposed by Faccio et al. [30] but much research still needs to be carried out concerning safety issues [9].

Although the companies in the survey by Land et al. [25] expressed that the sought after benefits of the ICR are both economical and ergonomic, the companies in this study argues that evaluating the outcome of the ICR application can be a challenge. One such challenge was that cycle times were not met in the ICR application. The slower cycle time is an issue also identified in academia concerning ICR applications [30]. Nonetheless, ICR applications are considered to be robust providing controllable and repeatable performance [5].

From the interview study, it is indicated that lack of expertise was one of the issues when employing an ICR application. In Granlund et al. [24], it was emphasized that lack of expertise is a common issue when companies deal with automation in general. The ICR application add to the integration complexity because the companies are unable to specify the sought after capabilities of the ICR application together with the supplier.

In summary, Table 1 presents the combines experienced capabilities of the ICR compared to traditional robots found in this study. The capabilities presented in this paper builds on the work of Hentout et al. [4], with the addition of providing empirical evidence for some of the capabilities. 
Table 1. Perceived ICR application capabilities compared to traditional robot applications

\begin{tabular}{|c|c|c|c|}
\hline Type & Flexibility & Safety & Applicability \\
\hline ICR & $\begin{array}{l}\text { - Some brands easy to } \\
\text { program } \\
\text { - Lightweight design } \\
\text { promote portability, in } \\
\text { some cases }\end{array}$ & $\begin{array}{l}\text { - Used in Fenceless } \\
\text { applications } \\
\text { - Inherent safety } \\
\text { - Varying Uncertainty in } \\
\text { safety assessments }\end{array}$ & $\begin{array}{l}\text { - Slow in collaborative } \\
\text { application } \\
\text { - Can work in close } \\
\text { proximity to humans and } \\
\text { support with repetitive } \\
\text { and precise tasks }\end{array}$ \\
\hline Traditional & $\begin{array}{l}\text { - Programing experienced } \\
\text { as more demanding } \\
\text { - Commonly immovable } \\
\text { due to fenced } \\
\text { application and robust } \\
\text { design }\end{array}$ & $\begin{array}{l}\text { - Fenced application } \\
\text { - Inherent safety limited } \\
\text { - Less uncertainty in safety } \\
\text { assessments due to humans } \\
\text { outside fence. }\end{array}$ & $\begin{array}{l}\text { - Are fast in fences } \\
\text { applications } \\
\text { - Limited, although } \\
\text { possible, use in } \\
\text { collaboration }\end{array}$ \\
\hline
\end{tabular}

This paper provides insight on the practitioner's sought after capabilities in ICR applications and an indication that there is still several capabilities that have not been fully employed in industry. For future research, a multiple case study with the users of ICR applications could provide deeper knowledge on hinders and enablers when integrating an ICR application. By doing so, knowledge can be added to the field of advanced manufacturing technology integration. This work was founded by the Knowledge Foundation (KKS) via the research school ARRAY.

\section{References}

[1] S. Kotha and P. M. Swamidass, Strategy, advanced manufacturing technology and performance : empirical evidence from U.S manufacturing firms, Journal of Operations Management, vol. 18, no. 3, 2000, pp. 257-277.

[2] B. Siciliano and O. Khatib, Springer handbook of robotics, 2nd ed., vol. 46, no. 06. Berlin Heidelberg, 2016.

[3] Å. Fasth, J. Stahre, and K. Dencker, Level of Automation Analysis in Manufacturing Systems, W, Karwowski and G, Salvendy, in Advances in human factors, ergonomics, and safety in manufacturing and service industries, CRC Press Taylor \& Francis Group, No. May, Boca Raton, FL, 2010, pp. 233-242.

[4] A. Hentout, M. Aouache, A. Maoudj, and I. Akli, Human - robot interaction in industrial collaborative robotics : a literature review of the decade 2008-2017, Advanced Robotics, vol. 1864, no. 33:15-16, 2019, pp. 764-799.

[5] L. Wang et al., Symbiotic human-robot collaborative assembly, CIRP Annals, vol. 68, no. 2, 2019, pp. 701-726.

[6] G. Michalos, S. Makris, N. Papakostas, D. Mourtzis, and G. Chryssolouris, Automotive assembly technologies review: challenges and outlook for a flexible and adaptive approach, CIRP Journal of Manufacturing Science and Technology, vol. 2, no. 2, 2010, pp. 81-91.

[7] J. Krüger, T. K. Lien, and A. Verl, Cooperation of human and machines in assembly lines, CIRP Annals - Manufacturing Technology, vol. 58, no. 2, 2009, pp. 628-646.

[8] P. Tsarouchi et al., A Decision Making Framework for Human Robot Collaborative Workplace Generation, 6th CIRP Conference on Assembly Technologies and Systems (CATS), Gothenburg, 2016, vol. 44, 2016, pp. 228-232.

[9] V. Villani, F. Pini, F. Leali, and C. Secchi, Survey on human-robot collaboration in industrial settings: Safety, intuitive interfaces and applications, Mechatronics, vol. 55, no. February, 2018, pp. $248-266$.

[10] IFR, 2017, Executive Summary World Robotics 2017 Industrial Robots, Accessed: 08-Aug-2019. [Online].Available: https://ifr.org/downloads/press/Executive_Summary_WR_2017_Industrial_Robots.pdf

[11] R. Burger and M. Barbagelata, 2019, Collaborative Robots: Industry Report, Accessed: 08-Aug- 
2019. [Online]. Available: http://www.woodsidecap.com/wcp-proudly-releases-the-collaborativerobots-industry-report/.

[12] V. Braun and V. Clarke, Using thematic analysis in psychology Using thematic analysis in psychology, Qualitative research in psychology, vol. 3, no. 2, 2008, pp. 77-101.

[13] E. Garcia, M. A. Jimenez, P. G. De Santos, and M. Armada, The evolution of robotics research, IEEE Robotics and Automation Magazine, vol. 14, no. 1, 2007, pp. 90-103.

[14] Robots And Robotic Devices - Safety Requirements For Industrial Robots - Part 1: Robots, 10218-1, 20-07-2011.

[15] I. Aaltonen, T. Salmi, and I. Marstio, Refining levels of collaboration to support the design and evaluation of human-robot interaction in the manufacturing industry, Procedia CIRP, vol. 72, 2018, pp. 93-98.

[16] Machine Directive. 2006/42/EC. 24-86, 09-06-2006.

[17] Robots and Robotic devices - Collaborative Robots, 15066:2016, 04-02-2016.

[18] V. Gopinath, F. Ore, S. Grahn, and K. Johansen, Safety-Focussed Design of Collaborative Assembly Station with Large Industrial Robots, Procedia Manufacturing, vol. 25, 2018, pp. 503-510.

[19] A. Khalid, P. T. Kirisci, and Z. Ghrairi, Safety Requirements in Collaborative Human Robot Cyber Physical Systems, in: Dynamics in logistics, Bremen, 2017, pp. 41-51.

[20] W. Bauer, M. Bender, M. Braun, P. Rally, and O. Scholtz, Lightweight robots in manual assembly best to start simply!, Frauenhofer-Institut für Arbeitswirtschaft und Organisation IAO. Stuttgart, 2016.

[21] F. Gil-Vilda, A. Sune, J. A. Yagüe-Fabra, C. Crespo, and H. Serrano, Integration of a collaborative robot in a U-shaped production line: a real case study, Procedia Manufacturing, vol. 13, 2017, pp. $109-115$.

[22] K. R. Guerin, C. Lea, C. Paxton, and G. D. Hager, A Framework for End-User Instruction of a Robot Assistant for Manufacturing, in: 2015 IEEE International Conference on Robotics and Automation (ICRA), Seattle, 2015, pp. 6167-6174.

[23] C. Paxton, A. Hundt, F. Jonathan, K. Guerin, and G. D. Hager, CoSTAR: Instructing Collaborative Robots with Behavior Trees and Vision, in: 2017 IEEE International Conference on Robotics and Automation (ICRA), Singapore, 2017, pp. 564-571.

[24] A. Granlund and M. Jackson, Managing Automation Development Projects - A Comparison of Industrial Needs and Existing Theoretical Support, in: 23rd International Conference on Flexible Automation and Intelligent Manufacturing, Porto, 2013, pp. 761-774.

[25] L. Land, A. Syberfeldt, A. Torgny, and A. Vallhagen, Virtual Human-Robot Collaboration : The Industry' s Perspective on Potential Applications and Benefits, in: 17th International Conference on Manufacturing Research, incorporating the 34th National Conference on Manufacturing Research, September 10-12, Belfast, 2019, pp. 161-166.

[26] P. Akella et al., Cobots for the automobile assembly line, in: Proceedings of the 1999 IEEE International Conference on Robotics \& Automation, Detroit, 1999, pp. 728-733.

[27] B. Schmidt and L. Wang, Depth camera based collision avoidance via active robot control, Journal of Manufacturing Systems, vol. 33, no. 4, 2014, pp. 711-718.

[28] F. Steinmetz and R. Weitschat, Skill parametrization approaches and skill architecture for humanrobot interaction, in: IEEE International Conference on Automation Science and Engineering (CASE), Fort Worth, 2016, pp. 280-285.

[29] S. Kock et al., Robot concept for scalable, flexible assembly automation: A technology study on a harmless dual-armed robot, in: Proceedings - 2011 IEEE International Symposium on Assembly and Manufacturing, Tempere, 2011, pp. 1-5.

[30] M. Faccio, M. Bottin, and G. Rosati, Collaborative and traditional robotic assembly: a comparison model, The International Journal of Advanced Manufacturing Technology, Vol. 102, no. 5-8, 2019, pp. 1355-1372. 